\title{
Cell-autonomous Toxoplasma killing program requires Irgm2 but not its microbe vacuolar localization
}

\author{
Ariel Pradipta ${ }^{1,2}$, Miwa Sasai ${ }^{1,2}$, Kou Motani $^{4}$, Ji Su Ma ${ }^{1}$, Youngae Lee ${ }^{2}$, Hidetaka Kosako ${ }^{4}$, Masahiro Yamamoto $^{1,2,3}$ (B)
}

\begin{abstract}
Interferon-inducible GTPases, such as immunity-related GTPases (IRGs) and guanylate-binding proteins (GBPs), are essential for cellautonomous immunity against a wide variety of intracellular pathogens including Toxoplasma. IRGs comprise regulatory and effector subfamily proteins. Regulatory IRGs Irgm1 and Irgm3 play important roles in anti-Toxoplasma immunity by globally controlling effector IRGs and GBPs. There is a remaining regulatory IRG, called Irgm2, which highly accumulates on parasitophorous vacuole membranes (PVMs). Very little is known about the mechanism of the unique localization on Toxoplasma PVMs. Here, we show that Irgm2 is important to control parasite killing through recruitment of Gbp1 and Irgb6, which does not require Irgm2 localization at Toxoplasma PVMs. Ubiquitination of Irgm2 in the cytosol, but not at the PVM, is also important for parasite killing through recruitment of Gbp1 to the PVM. Conversely, PVM ubiquitination and p62/Sqstm1 loading at later time points post-Toxoplasma infection require Irgm2 localization at the PVM. Irgm2-deficient mice are highly susceptible to Toxoplasma infection. Taken together, these data indicate that Irgm2 selectively controls accumulation of anti-Toxoplasma effectors to the vacuole in a manner dependent or independent on Irgm2 localization at the Toxoplasma PVM, which mediates parasite killing.
\end{abstract}

DOI 10.26508/lsa.202000960 | Received 16 November 2020 | Revised 17 May 2021 | Accepted 20 May 2021 | Published online 2 June 2021

\section{Introduction}

Pathogen infection activates a series of immune responses in healthy mammalian hosts. Innate immunity initially detects pathogen-derived components through germline-encoded receptors, such as Toll-like receptors, which induces a proinflammatory cytokine called interleukin12 (Hunter \& Remington, 1995; Yarovinsky \& Sher, 2006). This cytokine subsequently stimulates the acquired type I immune response through which naive $\mathrm{CD}^{+}$and $\mathrm{CD} 8^{+} \mathrm{T}$ cells differentiate into pathogen-derived antigen-specific Th1 cells and cytotoxic T cells with the help of antigenpresenting cells (Gazzinelli et al, 1991). These T-cell subsets together with natural killer cells robustly produce IFN- $y$ to activate cellautonomous immunity that eliminates various vacuolar pathogens, including a major human and animal protozoan Toxoplasma gondii (Sturge \& Yarovinsky, 2014).

IFN- $\gamma$ stimulates production of hundreds of IFN- $\gamma$-stimulated proteins such as indole 2,3-deoxygenase (IDO), inducible nitric oxide synthase (iNOS), and IFN-inducible GTPases. In the infected cells, IDO and iNOS inhibit T. gondii proliferation by depleting tryptophan and arginine, respectively (Hunter \& Sibley, 2012). IFNinducible GTPases, such as p47 immunity-related GTPases (IRGs) and p65 guanylate-binding proteins (GBPs), localize at membranes of $T$. gondii parasitophorous vacuole (PV) in which the parasite sequesters host nutrients and proliferates efficiently (Kim et al, 2012). Accumulation of IRGs and GBPs eventually disrupts the $T$. gondii parasitophorous vacuole membrane (PVM) to kill the pathogen (Martens \& Howard, 2006; Saeij \& Frickel, 2017). In mice, the IRG protein family comprises three regulator proteins (Irgm1, Irgm2, and Irgm3) and >20 effector proteins (Bekpen et al, 2005; Pilla-Moffett et al, 2016). Effector IRG proteins harbor a universally conserved $\mathrm{GX}_{4} \mathrm{GKS}$ sequence in the nucleotide-binding motif (G1), which enables binding to both GDP and GTP (Uthaiah et al, 2003; Bekpen et al, 2005). Conversely, the G1 motif of regulator IRG proteins possess a GX $\mathrm{K}_{4} \mathrm{GMS}$ sequence that mainly binds to GDP by which effector IRGs are maintained in an inactive state and their activation is prevented (Bekpen et al, 2005; Hunn et al, 2008). Such negative regulation might be important for protection of host endomembranes, because Irgm1 and Irgm3 localize at the host Golgi apparatus and ER, respectively (Martens et al, 2004; Hunn et al, 2008; Haldar et al, 2013). Lack of Irgm1 and Irgm3 results in the formation of nucleotide-dependent cytoplasmic aggregates that are caused by premature GTP binding and activation of effector IRGs, which leads to severely impaired loading of effector IRGs and GBPS on the T. gondii PVM and dismantling cell-intrinsic immunity (Taylor et al, 2000; Maric-Biresev et al, 2016). Thus, IRG and GBPdependent cell-intrinsic immunity against vacuolar pathogens is well known to be globally controlled by Irgm1 and Irgm3. In addition, the localization of IRGs and GBPs is regulated by autophagy-related proteins (ATGs) in a manner independent of autophagy (Zhao et al, 2008; Yamamoto et al, 2012; Ohshima et al, 2014; Park et al, 2016; Sasai et al, 2017). The rest of a member of a regulator IRG protein is

\footnotetext{
${ }^{1}$ Department of Immunoparasitology, Research Institute for Microbial Diseases, Osaka University, Osaka, Japan ${ }^{2}$ Laboratory of Immunoparasitology, WPI Immunology Frontier Research Center, Osaka University, Osaka, Japan ${ }^{3}$ Division of Microbiology and Immunology, Center for Infectious Disease Education and Research, Osaka University, Osaka, Japan ${ }^{4}$ Division of Cell Signaling, Fujii Memorial Institute of Medical Sciences, Tokushima University, Tokushima, Japan
} 
Irgm2, which localizes to the T. gondii PVM as well as the host Golgi apparatus (Hunn et al, 2008). However, the physiological role of Irgm2 and particularly the molecular mechanism of T. gondii PVM localization remain uncertain.

Effector IRG proteins, such as Irga6, Irgb6, and Irgb10, accumulate on the T. gondii PVM in a hierarchical manner (Khaminets et al, 2010). Irgb6 initially detects the T. gondii PVM via its phospholipid binding and leads to subsequent loading of Irga6 and Irgb10 (Lee et al, 2020), recruitment of GBPs that comprise 11 members in mice (Kresse et al, 2008). Some effector IRG proteins mutually control the localization of GBPs. Irgb6 controls accumulation of Gbp1 on the PVM and vice versa (Selleck et al, 2013) and Gbp2 regulates Irga6 loading on the PVM (Ohshima et al, 2015). Accumulation of effector IRGs and GBPs in turn damages the PVM (Martens et al, 2005; Kravets et al, 2016), which leads to PVM ubiquitination, followed by p62/ Sqstm1 coating (Haldar et al, 2015; Lee et al, 2015). One study has demonstrated that ubiquitination of T. gondii PVM is important for parasite killing (Haldar et al, 2015). A subsequent recent study has demonstrated roles of TRAF6 and TRAF2 in PVM ubiquitination and TRAF6-dependent killing of $T$. gondii in IFN- $y$-primed mouse fibroblasts (Mukhopadhyay et al, 2020). Conversely, another study has shown that ubiquitination and p62 coating is dispensable for $T$. gondii killing (Lee et al, 2015). Thus, the biological significance of IFN- $\gamma$-inducible T. gondii PVM ubiquitination is a matter of debate. Moreover, the ubiquitin substrate(s) on T. gondii PVM is unclear.

Here, we aimed to determine the role of Irgm2 in cell-intrinsic immune responses to $T$. gondii. We found that Irgm2 specifically participates in Irgb6 and Gbp1-mediated parasite killing by regulating their accumulation on the T. gondii PVM. Furthermore, a cysteine residue in the C-terminus of Irgm2 determines its localization on the T. gondii PVM, which is important for prolonged loading of ubiquitin and p62 on the PVM but not for Irgb6- and Gbp1-mediated parasite killing. Moreover, we found that ubiquitinated Irgm2 is required for Gbp1-mediated T. gondii killing but may not localize at the PVM. Finally, we found that Irgm2-deficient mice are highly susceptible to T. gondii infection. Collectively, these data demonstrate that IFN$\gamma$-induced cell-autonomous immunity against $T$. gondii is regulated in a manner dependent or independent on Irgm2 PVM localization.

\section{Results}

\section{Irgm2-deficient cells are defective for recruitment of T. gondii clearance-related effectors}

To assess the role of Irgm2 in anti-T. gondii immune responses, we generated Irgm2-deficient mice by CRISPR/Cas9 genome editing (Fig S1A and B). Anti-Irgm2 did not detect Irgm2 proteins in MEFs from Irgm2-deficient mice (Fig S1A and B). We first obtained BMDMs, BMDCS, and MEFs from wild-type and Irgm2-deficient mice, and compared the IFN- $\gamma$-induced reduction of $T$. gondii parasite numbers in the cells (Fig 1A). We found that Irgm2-deficient BMDMs, BMDCs, and MEFs were defective in IFN- $y$-induced reduction of $T$. gondii numbers in comparison with wild-type cells (Fig 1A). However, the magnitude of the defect in single Irgm2-deficient cells was not as severe as Irgm1/Irgm3 double KO (DKO) or Irgm1/Irgm2/Irgm3 triple knockout (TKO) cells (Figs 1A and S1C), which indicates that Irgm2 as well as Irgm1 and Irgm3 contributes to the IFN- $y$-mediated cellautonomous response to $T$. gondii. Recruitment of effector IRGs and GBPs has been extensively studied as important host cell-intrinsic events for T. gondii killing. We compared localization of effector IRGs, such as Irga6 and Irgb6, and GBPS, such as Gbp1 and Gbp2, on the T. gondii PVM in wild-type and Irgm2-deficient cells (Fig 1B and C). We confirmed that IRG and GBP effector proteins were expressed at comparable levels in wild-type and Irgm2-deficient cells (Fig 1D). Taken together, these results demonstrate that Irgm2 deficiency impairs T. gondii killing activity with selectively decreases recruitment of Irgb6 and Gbp1 on the PVM.

\section{The GMS configuration of the Irgm2 GTPase domain is essential for $T$. gondii clearance activity}

We next explored the molecular mechanisms by which Irgm2 regulated the IFN- $y$-induced cell-autonomous immune response. Irgm2 possesses an N-terminal GTPase domain (Figs 2A and S1D). It has been previously shown that the GKS configuration in the N-terminal GTPases of Irga6 and Irgb6 is important for their recruitment function to the $T$. gondii PVM (Hunn et al, 2008; Papic et al, 2008; Lee et al, 2020). Irgm2 harbors a GMS sequence in its GTPase domain, in which the mutated methionine is a part of the unconventional GMS P-loop sequence of immunity-related GTPase family M (IRGM) proteins (Bekpen et al, 2005). Therefore, we examined the role of the Irgm2 GTPase domain. A point mutant, in which the methionine at position 77 in the GTPase domain of Irgm2 was substituted with alanine (Irgm2 M77A), may disrupt nucleotide (GDP) binding and hence cause non-functionality (Hunn et al, 2008). We reconstituted wild-type and M77A Irgm2 in Irgm2-deficient MEFs for IFN- $\gamma$-induced reduction of $T$. gondii numbers and recruitment of Irgb6 and Gbp1 (Fig 2B and C). We reconstituted Irgm2-deficient MEFs with wild-type Irgm2 and the M77A mutant, and confirmed similar expression (Fig 2B). When IFN- $\gamma$-induced killing activity was examined, Irgm2-deficient MEFs reconstituted with wild-type Irgm2 were able to recover the killing activity (Fig $2 \mathrm{C}$ ). In sharp contrast, Irgm2 KO MEFs that expressed the M77A mutant were not able to restore this killing activity (Fig 2C). Furthermore, reconstitution of wild-type Irgm2 in Irgm2deficient MEFs recovered recruitment of Gbp1 and Irgb6, whereas that of the M77A mutant did not (Fig 2D-F). Wild-type Irgm2, but not the M77A mutant, was found on the T. gondii PVM (Fig 2D and G) as described previously (Martens \& Howard, 2006; Zhao et al, 2010). Taken together, these results demonstrate that the GMS configuration of Irgm2 is essential for the cell-autonomous immune function of Irgm2.

\section{Regulatory IRGs and ATG proteins are dispensable for Irgm2 localization on the T. gondii PVM}

Next, we examined the regulatory mechanism of Irgm2 localization on the T. gondii PVM. As reported previously (Haldar et al, 2013), localization of Irgb6 and Gbp1 was severely impaired in cells that lacked Irgm1 and Irgm3 (Fig S2A-D). Surprisingly, the localization of Irgm2 was normal in Irgm1/Irgm3 double-deficient cells (Fig 3A and B), which suggests that these regulatory IRGs do not control Irgm2 localization on the T. gondii PVM. In addition to regulatory IRGs, autophagy proteins, such as Atg3 and Atg7, are essential for correct targeting of effector IRGs to the T. gondii PVM (Choi et al, 2014; Ohshima et al, 2014; Sasai et al, 2017). However, cells that lacked Atg3 


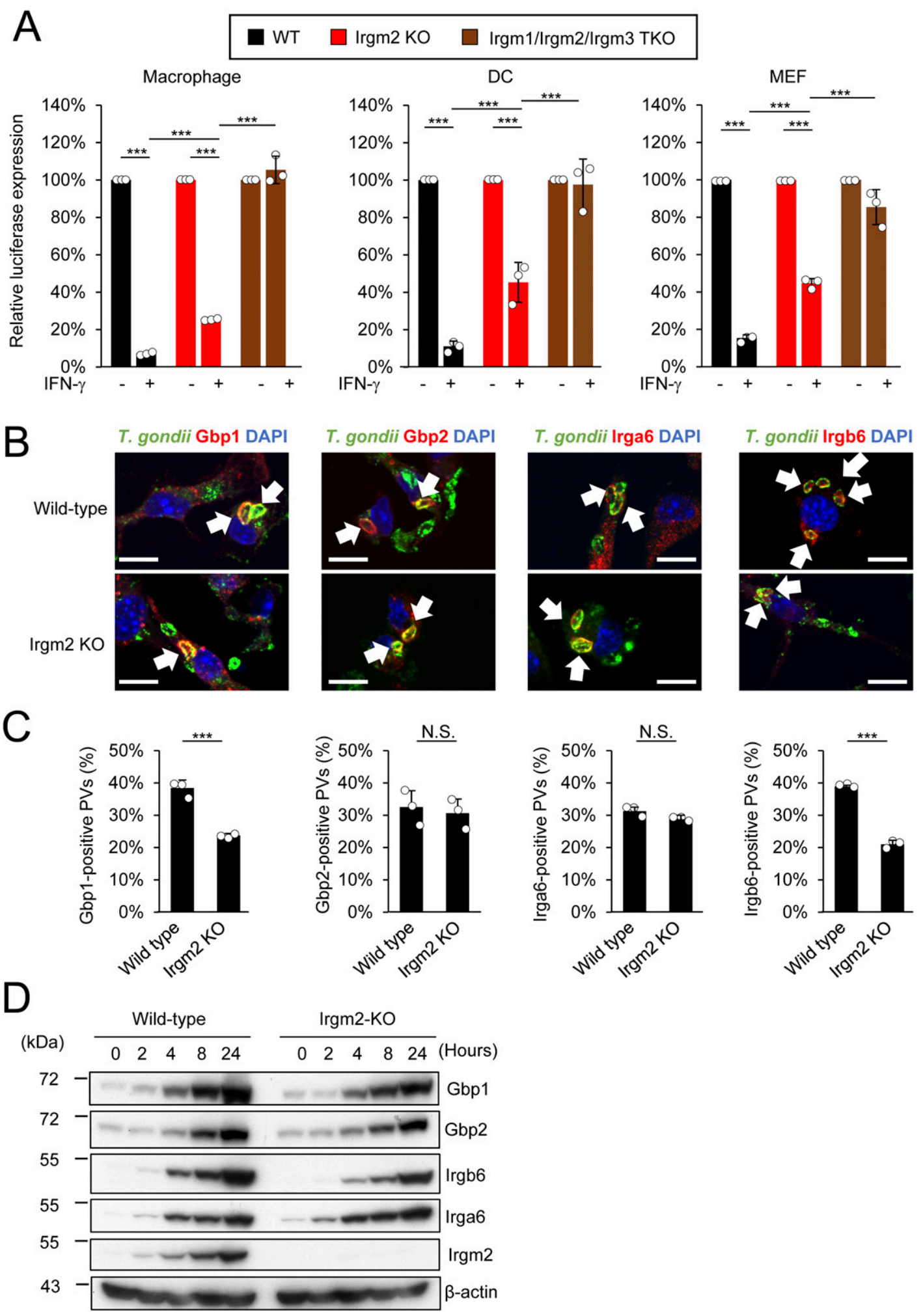

Figure 1. Irgm2 selectively controls IFN-inducible GTPase-dependent cell-autonomous immunity.

(A) Toxoplasma gondii survival rate in BMDM, BMD-DC, and MEF with IFN- $y$ stimulation relative to those without IFN- $y$ treatment by luciferase analysis at $24 \mathrm{~h}$ post infection. (B) Confocal microscope images to show the localization of various effectors (red) including Gbp1, Gbp2, Irga6, and Irgb6 to T. gondii parasitophorous vacuole (green) and DAPI (blue) at $4 \mathrm{~h}$ post infection in IFN- $y$ treated MEFs indicated on the sides. (C) Recruitment percentages of Gbp1, Gbp2, Irga6, and Irgb6. (D) Western blot image of indicated protein expression in WT and Irgm2 KO MEFs at indicated hours post IFN- $\gamma$ activation. All graphs show the mean \pm SEM in three independent experiments. All images are representative of three independent experiments. N.D., not detected; ${ }^{\star} P<0.05,{ }^{\star \star} P<0.01$, ${ }^{\star \star \star} P<0.001$. Difference in $T$. gondii inhibition activity between IFN- $y$-activated versus nonactivated was subjected to two-way ANOVA, with Tukey's multiple comparisons test to analyze the difference between genotypes. Effector recruitment comparison between only two genotypes analyzed with Mann-Whitney test. White arrows indicate recruitment of effector on $T$. gondii parasitophorous vacuole. Scale bars on microscope images represent $10 \mu \mathrm{m}$. 

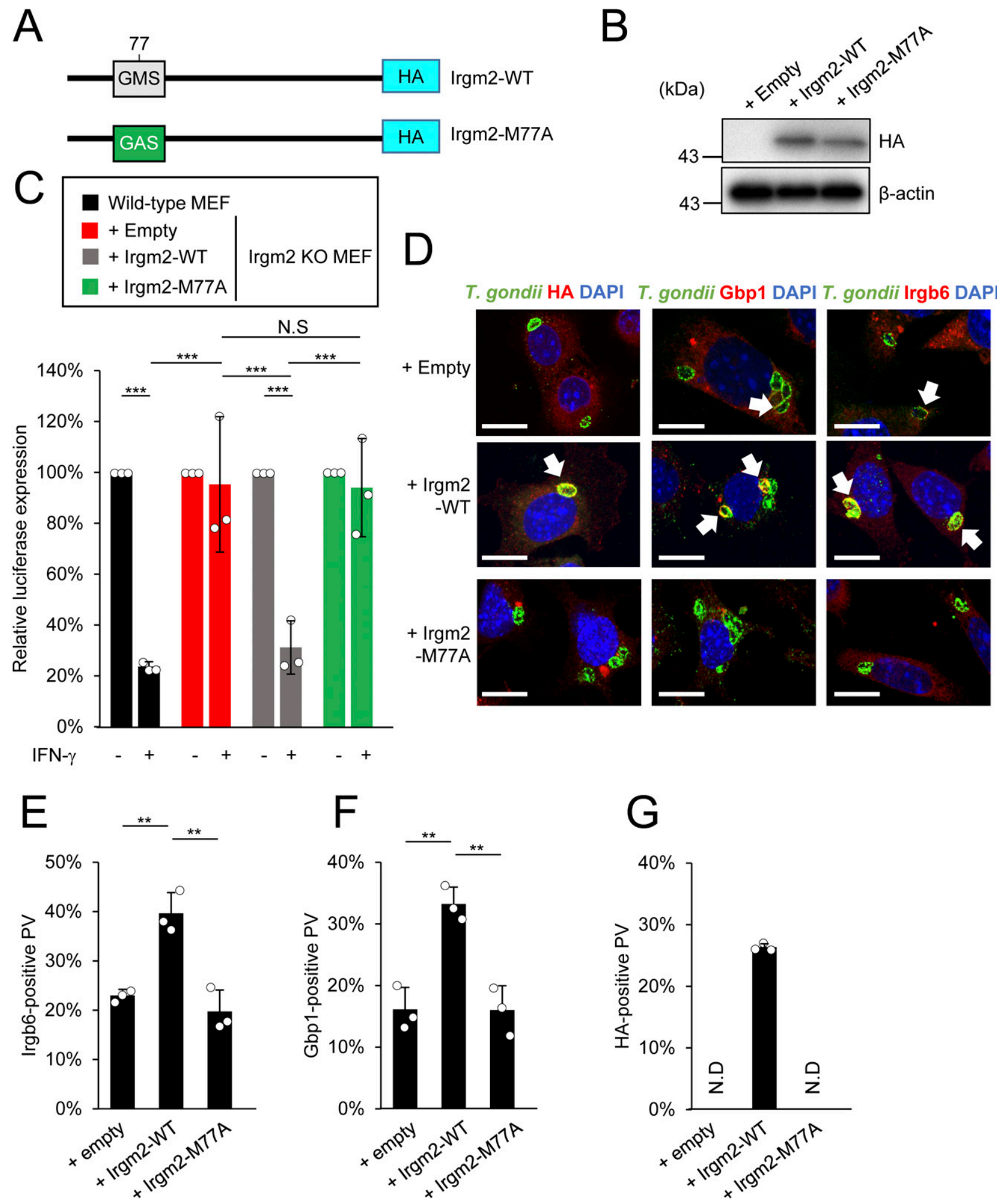

Figure 2. The guanine nucleotide binding moiety is essential for Irgm2-dependent anti-Toxoplasma gondii programs.

(A) Schematic overview of the substitution mutation site on the Irgm2 sequence. (B) Western blot image to detect stably expressed Irgm2 protein after retroviral transfection and puromycin selection. (C) T. gondii survival rate in the indicated Irgm2 reconstitution in Irgm2 KO MEFs with IFN- $\gamma$ stimulation relative to those without IFN- $\gamma$ treatment by luciferase analysis at $24 \mathrm{~h}$ post infection. (D) Confocal microscope images to show the localization of Irgm2-HA, Gbp1, and Irgb6 (red) to T. gondii parasitophorous vacuole (green), and DAPI (blue) at $4 \mathrm{~h}$ post infection in IFN- $\gamma$-treated Irgm2-KO MEFs reconstituted with Irgm2-WT and Irgm2-M77A. (E, F, G) Recruitment percentages of Irgb6 (E), Gbp1 (F), HA (G). All graphs show the mean \pm SEM in three independent experiments. All images are representative of three independent experiments. N.D., not detected; ${ }^{\star} P<0.05$, ${ }^{\star \star} P<0.01,{ }^{\star \star \star} P<0.001$. Difference in T. gondii inhibition activity between IFN- $\gamma$-activated versus nonactivated was subjected to two-way ANOVA, with Tukey's multiple comparisons test to analyze the difference between genotypes. Effector recruitment comparison between genotypes applied one-way ANOVA (Tukey's multiple comparisons test). White arrows indicate recruitment of effector on T. gondii parasitophorous vacuole. Scale bars on microscope images represent $10 \mu \mathrm{m}$. 
A
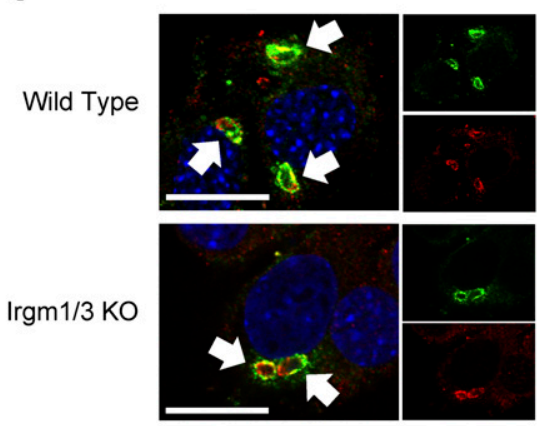

Irgb6 KO
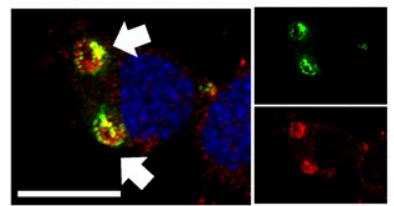

Gbp1 KO

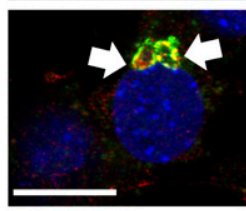

T. gondii Irgm2 DAPI

Atg3 KO
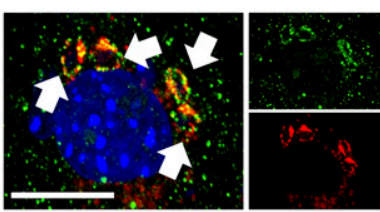

$\operatorname{Atg} 7 \mathrm{KO}$

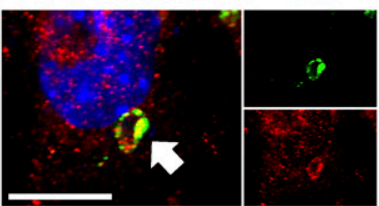

Atg9 $\mathrm{KO}$
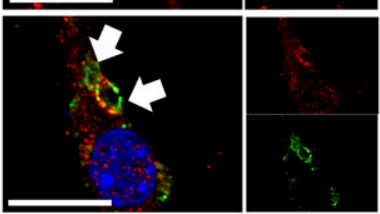

Atg14 KO

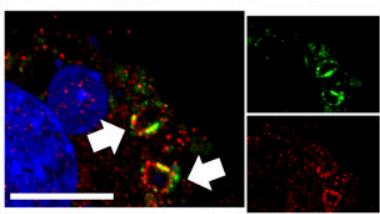

Figure 3. Irgm2 recruitment to Toxoplasma gondi parasitophorous vacuole (PV) is independent of Irgm1/Irgm3 and Atg proteins.

(A) Confocal microscope images to show the localization of Irgm2 (red) to T. gondii PV (green) and DAPI (blue) at $4 \mathrm{~h}$ post infection in IFN- $y$ treated indicated MEFs. (B) Percentages of endogenous Irgm2 recruitment to $T$. gondii $P V$ in indicated cells are shown. All graphs show the mean \pm SEM in three independent experiments. All images are representative of three independent experiments. N.D., not detected. White arrows indicate recruitment of effector on T. gondii PV. Scale bars on microscope images represent $10 \mu \mathrm{m}$. All graphs show the mean \pm SEM in three independent experiments. All images are representative of three independent experiments. N.D., not detected; ${ }^{*} P<0.05,{ }^{* *} P<0.01,{ }^{* * *} P<0.001$. Effector recruitment comparison between genotypes applied one-way ANOVA (Tukey's multiple comparisons test). White arrows indicate recruitment of effector on T. gondii PV. Scale bars on microscope images represent $10 \mu \mathrm{m}$.

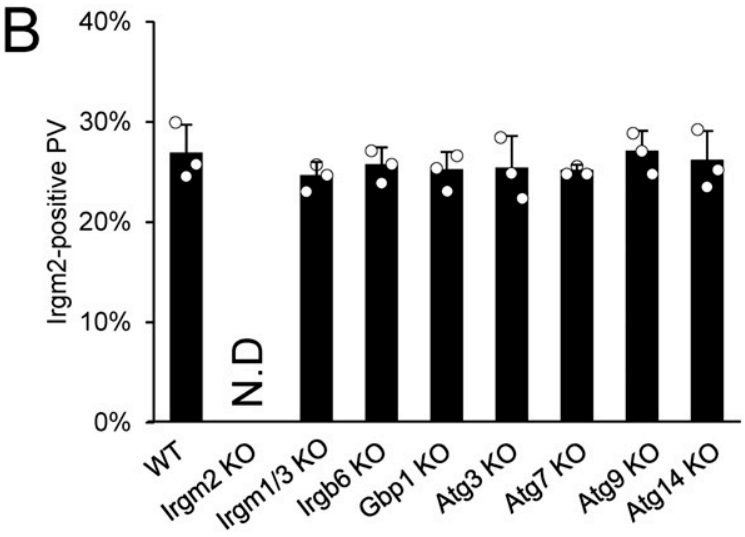

and Atg7 as well as Atg9 or Atg14 displayed normal Irgm2 recruitment of the T. gondii PVM (Fig $3 \mathrm{~A}$ and $\mathrm{B}$ ), which indicates that Atg proteins are dispensable for Irgm2 localization on the T. gondii PVM. GBPs and effector IRGs mutually regulate localization of each other (Yamamoto et al, 2012; Selleck et al, 2013; Ohshima et al, 2015; Saeij \& Frickel, 2017; Lee et al, 2020). Although localization of Irgb6 or Gbp1 on the T. gondii PVM was affected by Gbp1 or Irgb6 deficiency (Fig S2A-D), Irgm2 localization was normal in Irgb6- or Gbp1-deficient cells (Fig 3A and B), which demonstrated that regulatory IRGs and Atg proteins are not involved in Irgm2 localization on the T. gondii PVM.

\section{A cysteine in the $C$ terminus of Irgm2 controls localization on the T. gondii PVM}

We further examined the molecular mechanism by which Irgm2 localizes at the T. gondii PVM. Irgb6, Irgb10, and Irgm1 possess an amphipathic helix in their $\mathrm{C}$ terminus called $\alpha \mathrm{K}$, which directs binding to the target membrane (Martens et al, 2004; Tiwari et al, 2009; Man et al, 2016; Lee et al, 2020). Localization of Irgm1, which is most homologous to Irgm2, at the host mitochondrial membrane was abolished by introducing mutations in a tight cluster of the cysteine near the $C$ terminus of Irgm1, which is immediately adjacent to the $\alpha \mathrm{K}$ region (Henry et al, 2014). When we searched for such potential cysteine residues in the corresponding $C$ terminus of Irgm2, we found a cluster of cysteines (aa357 and aa358) adjacent to the $\alpha \mathrm{K}$ (aa336-aa353) (Figs 4A and S1D). To investigate whether the cysteine residues are involved in Irgm2 targeting the T. gondii PVM, we generated Irgm2 C357A or C358A point mutants (Fig 4A), reconstituted them in Irgm2 KO MEFs (Fig 4B), and examined their localization in T. gondii-infected or uninfected cells (Figs $4 C$ and D and S3A). Although recruitment of C357A Irgm2 to the T. gondii PVM was comparable to that of wild-type Irgm2, it was of interest that the 
A

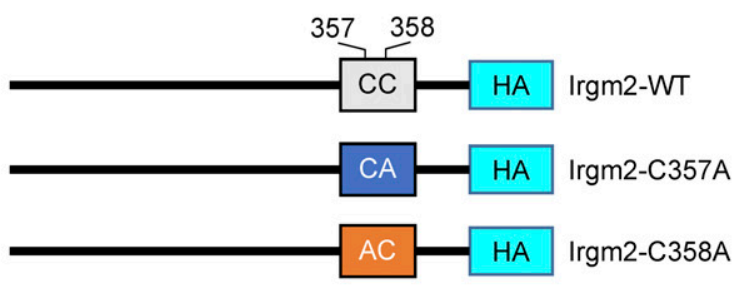

C
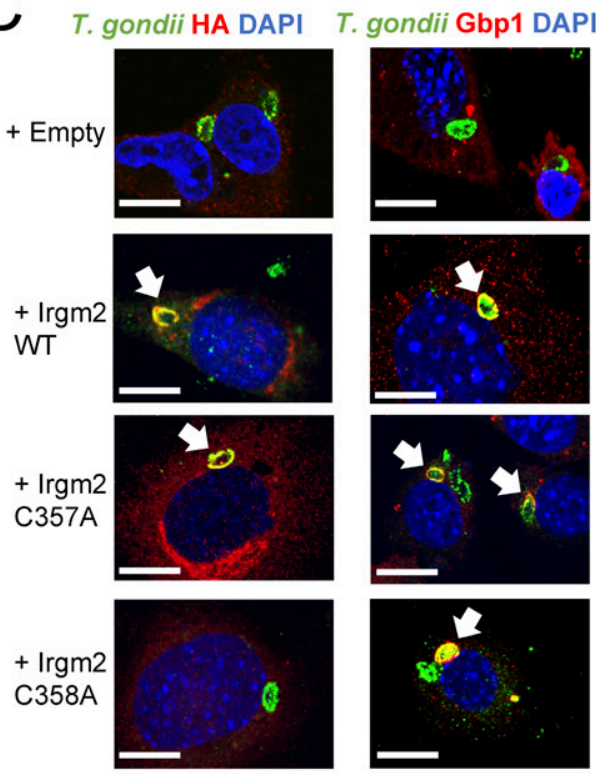

$\mathrm{E}$
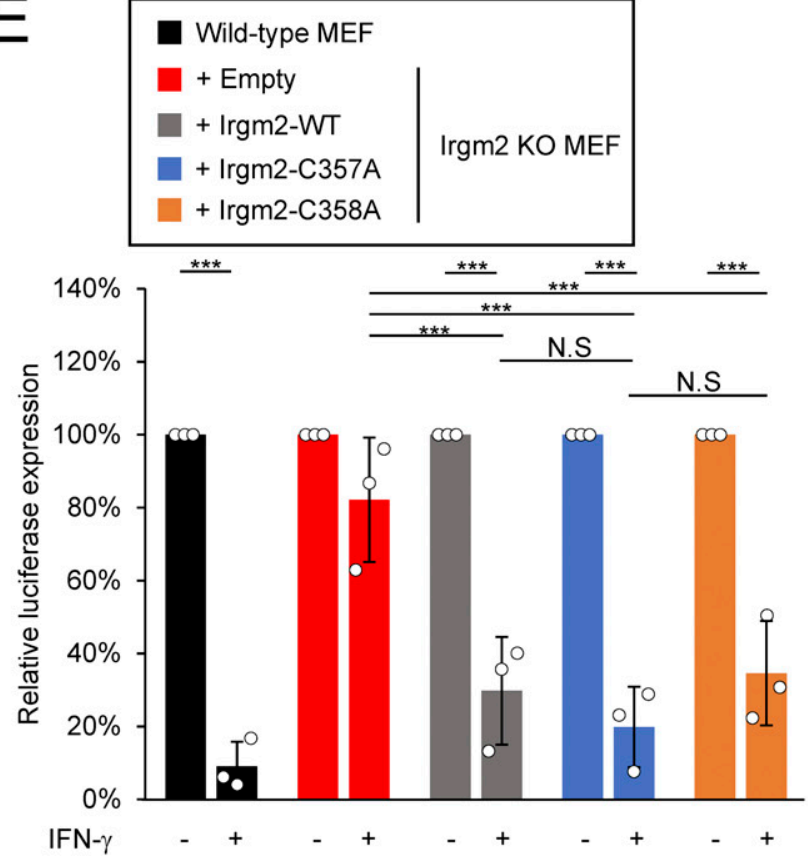

B
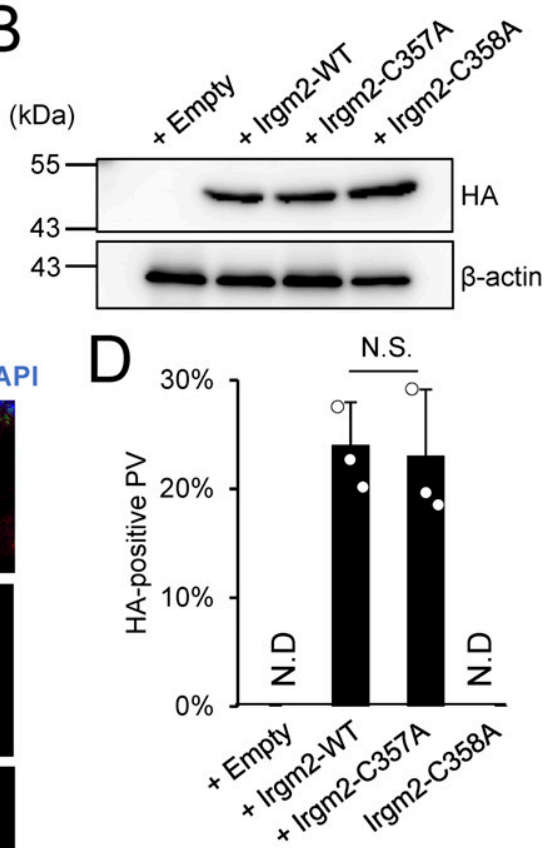

$\mathrm{F}$
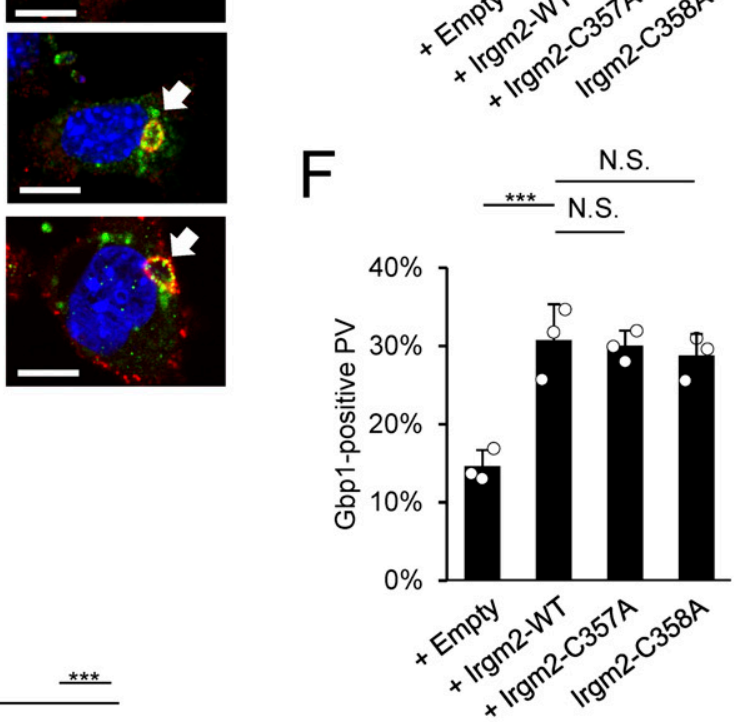

G

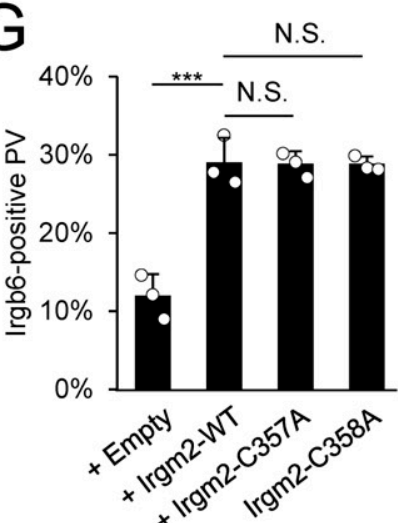

Figure 4. The C358A mutation in the C-terminus only affects its recruitment to Toxoplasma gondii parasitophorous vacuole (PV) but not the killing program. (A) Schematic overview of the substitution mutation site on the Irgm2 sequence. (B) Western blot image to detect stably expressed Irgm2 protein after retroviral. transfection and puromycin selection. (C) Confocal microscope images to show the localization of Irgm2-HA, Gbp1, and Irgb6 (red) to T. gondii PV (green), and DAPI (blue) at $4 \mathrm{~h}$ post infection in IFN- $\gamma$-treated Irgm2-KO MEFs reconstituted with indicated Irgm2. (D, F, G) Recruitment percentages of Irgm2-HA (D), Gbp1 (F), and Irgb6 (G). (E) T. gondii survival rate in the indicated Irgm2 reconstitution in Irgm2 KO MEFs with IFN- $\gamma$ stimulation relative to those without IFN- $\gamma$ treatment by luciferase analysis at $24 \mathrm{~h}$ post infection. All graphs show the mean \pm SEM in three independent experiments. All images are representative of three independent experiments. N.D., not 
C358A Irgm2 mutant did not accumulate on the T. gondii PVM (Fig 4C and D), which indicates that the Cys358 of Irgm2 is a determinant for localization on the T. gondii PVM. When localization of Irgm2 wildtype, C358A, and M77A mutants was tested in uninfected cells, the wild-type and M77A Irgm2 mutant were detected at the Golgi apparatus, whereas the C358A mutant was not (Fig S3A), which suggests that Cys358 is responsible for Irgm2 localization at the Golgi apparatus in uninfected cells. Next, we assessed whether Irgm2 localization on $T$. gondii is involved in IFN- $\gamma$-induced parasite clearance (Fig 4E). Surprisingly, reconstitution of the C358A mutant in Irgm2-deficient cells fully restored IFN- $\gamma$-induced T. gondii killing activity in a manner similar to that of wild-type Irgm2 (Fig 4E). Consistently, recruitment of Irgb6 and Gbp1 to the T. gondii PVM was recovered by reintroduction of the C358A mutant well as wild-type Irgm2 into Irgm2-deficient cells (Fig 4C, F, and G). Taken together, these data suggest that Irgm2 localization on the T. gondii PVM in infected cells as well as its accumulation at the Golgi apparatus in uninfected cells are determined by Cys358 at the C terminus, whereas it is not linked to Irgb6/Gbp1-mediated killing activity.

\section{Irgm2 localization on the T. gondii PVM is important for prolonged accumulation of p62 and ubiquitin}

Recruitment of p62, ubiquitin, Irgb6, and Gbp1 to the T. gondii PVM was compared between wild-type Irgm2- or the C358A-reconstituted or empty vector-transduced Irgm2-deficient cells in time-dependent manners (Fig 5A-D). When we compared ubiquitin loading and recruitment of Gbp1, Irgb6 and p62 among wild-type, Irgm2-deficient, and Irgm1/Irgm3 DKO cells, we found that ubiquitin loading and recruitment of these effectors were greatly reduced in Irgm1/Irgm3 DKO cells as reported previously (Lee et al, 2015). Compared with Irgm1/Irgm3 DKO cells, Irgm2-deficient cells were partially defective for loading of these effectors (Fig S3B). Despite no difference at $4 \mathrm{~h}$ postinfection, it was of note that the C358A Irgm2 mutant-reconstituted or empty vector-transduced Irgm2-deficient cells showed more rapid reduction in recruitment of p62 and ubiquitin at later time points ( 6 and $8 \mathrm{~h}$ postinfection) than wild-type Irgm2-reconstituted cells (Fig 5A and B). Conversely, there was no significant difference in recovery of Irgb6 and Gbp1 recruitment to the T. gondii PVM between wild-type Irgm2- and C358A mutant-reconstituted cells (Fig 5C and D), which suggests that Irgm2 localization on the T. gondii PVM is important for prolonged recruitment of p62 and ubiquitin on parasites. Taken together, these results demonstrate that Irgm2 localization on the T. gondii PVM is dispensable for parasite killing but indispensable for prolonged accumulation of p62 and ubiquitin on the PVM.

\section{Ubiquitination of Irgm2 in the cytosol is important for Gbp1 recruitment to the $T$. gondii PVM}

Next, we searched for potential sites of protein modifications, such as phosphorylation and ubiquitination, in Irgm2. We subjected Spot-tagged Irgm2 to mass spectrometric analyses and found that several lysine residues might be ubiquitinated (Figs 6A, S1D, and S4). When Flag-tagged wild-type Irgm2 was reconstituted in Irgm2-deficient cells that expressed $3 \times \mathrm{HA}$-tagged ubiquitin, the immune-precipitated Flag-tagged wild-type or C358A Irgm2 was heavily ubiquitinated, whereas the immune-precipitated KA mutant, in which all of the potential lysine residues were substituted to alanines, did not yield such ubiquitin smears (Fig 6B), which suggests that the lysine residues of Irgm2 are ubiquitination sites. Next, we assessed the significance of Irgm2 ubiquitination in IFN- $\gamma$-induced anti-T. gondii responses. Notably, reconstitution of the Irgm2 KA mutant in Irgm2-deficient cells only partially recovered parasite killing in comparison with that of wild-type Irgm2 (Fig 6C). Recruitment of Gbp1 and Irgb6 was evaluated in the reconstituted cells (Fig 6D-F). Accumulation of Irgb6 was comparable between wild-type Irgm2- and KA mutant-reconstituted cells (Fig 6D and F). Conversely, KA Irgm2-reconstituted cells showed significantly less Gbp1 recruitment to the T. gondii PVM than wild-type Irgm2-reconstituted cells (Fig 6D and E). When localization of the Irgm2 KA mutant was tested, the Irgm2 KA mutant was detected on the T. gondii PVM in a manner similar to wild-type Irgm2 (Fig 6D and G), which suggests that ubiquitination of Irgm2 is not involved in localization of itself on parasites. Next, we compared ubiquitination accumulation in Irgm2-deficient cells reconstituted with wild-type Irgm2 and the KA mutant (Fig 6H). Interestingly, ubiquitin accumulation in Irgm2 KA mutant-reconstituted cells was rapidly decreased at later time points in comparison with wild-type Irgm2 (Fig 6H). However, it remained unclear whether Irgm2 on PVM is ubiquitinated. Because Irgm1/Irgm3 DKO cells or Irgb6-deficient cells are severely defective for IFN- $y$-induced PVM ubiquitination (Lee et al, 2015, 2020), we compared recruitment of the Irgm2 KA mutant and ubiquitination on the PVM in cells that lacked Irgm1/Irgm3 or Irgb6 (Fig S5A-E). When wild-type Irgm2 and the KA mutant were ectopically expressed in Irgm1/Irgm2/Irgm3 TKO cells or Irgb6-deficient cells (Fig S5A), the Irgm2 KA mutant and wild-type Irgm2 were comparably detected on the PVM in either cell type (Fig S5B and C). In sharp contrast, IFN$\gamma$-induced ubiquitin loading was not detected in Irgm2 KA mutantreconstituted Irgm1/Irgm2/Irgm3 TKO cells or Irgb6-deficient cells (Fig S5D and E). Thus, the Irgm2 KA mutant normally localized on the PVM in cells defective for IFN-y-induced PVM ubiquitination, which suggests that Irgm2 on PVM may not be ubiquitinated. When interactions between Gbp1, Irgb6, and Irgm2 were examined, we found that wildtype or C358A Irgm2 were coprecipitated with both Gbp1 and Irgb6 (Fig $6 \mathrm{II}$. Conversely, the KA Irgm2 mutant only associated with Irgb6 but not with Gbp1 (Fig 6I). The M77A mutant did not interact with Irgb6 or Gbp1, which suggests that the capacity for Irgm2 binding to Gbp1 and Irgb6 correlates with recruitment of these effectors to the T. gondii PVM. Wild-type Irgm2 was localized at the Golgi apparatus in uninfected cells (Fig S3) (Zhao et al, 2010). However, the KA mutant as well as M77A or C358A mutants did not localize at the Golgi apparatus (Fig S3), which suggests the important role of ubiquitination on Irgm2 in Golgi localization. Taken together, these results indicate that ubiquitinated Irgm2 in the cytosol interacts with Gbp1 to promote recruitment of Gbp1 to the T. gondii PVM.

detected; ${ }^{*} P<0.05,{ }^{* *} P<0.01,{ }^{* * *} P<0.001$. Difference in T. gondii inhibition activity between IFN- $\gamma$-activated versus nonactivated was subjected to two-way ANOVA, with Tukey's multiple comparisons test to analyze the difference between genotypes. Effector recruitment comparison between genotypes applied one-way ANOVA (Tukey's multiple comparisons test). White arrows indicate recruitment of effector on $T$. gondii PV. Scale bars on microscope images represent $10 \mu \mathrm{m}$. 
A

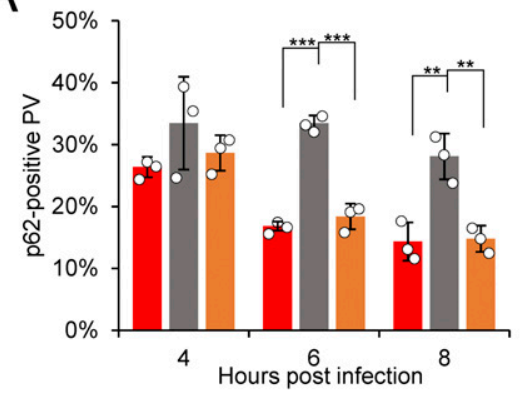

C

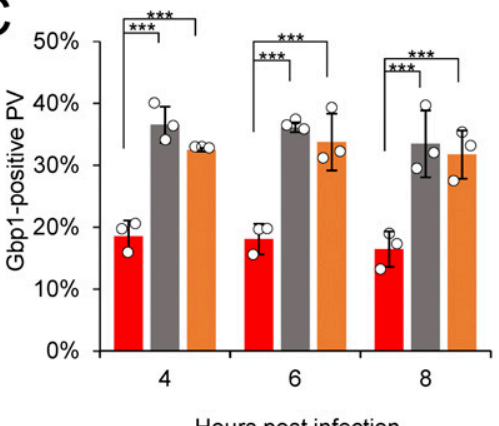

Hours post infection
B
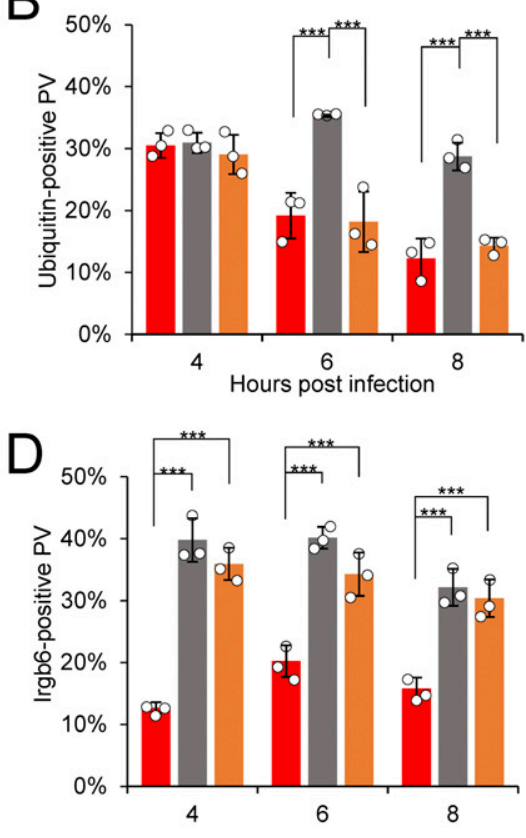

Hours post infection
Figure 5. The Cys358 of Irgm2 is indispensable for prolonged ubiquitin coating and $\mathbf{6 2}$ accumulation. (A, B, C, D) Percentages of recruitment of effectors p62 (A), ubiquitin (B), Gbp1 (C), and Irgb6 (D) on intracellular Toxoplasma gondii parasitophorous vacuole in IFN$\gamma$-stimulated Irgm2 KO MEFs reconstituted with the Irgm2 variations (empty vector: red, Irgm2 WT: grey, and Irgm2 C358A: orange) at indicated time points after $T$. gondii infection. All graphs show the mean \pm SEM in three independent experiments. All images are representative of three independent experiments. N.D., not detected; ${ }^{*} P<0.05,{ }^{* \star} P<0.01,{ }^{* * *} P<0.001$. Difference in T. gondii inhibition activity between IFN$\gamma$-activated versus nonactivated was subjected to two-way ANOVA, with Tukey's multiple comparisons test to analyze the difference between genotypes. Effector recruitment comparison between genotypes applied one-way ANOVA (Tukey's multiple comparisons test) or two-way ANOVA when time and genotype is considered.

\section{Irgm2-deficient mice are highly susceptible to $T$. gondii infection}

We finally examined the role of Irgm2 in the anti-T. gondii response in vivo (Fig 7A-C). Irgm2-deficient mice were infected with T. gondii that expressed luciferase by which the parasite dissemination could be measured by an in vivo imaging system (Fig 7A). At day 5 postinfection, Irgm2-deficient mice contained much higher luminescence emitted from luciferase-expressing T. gondii than wild-type mice (Fig $7 A)$. When the survival rate was assessed, Irgm2-deficient mice displayed high susceptibility to T. gondii (Fig 7B). In addition, the mortality of infected Irgm2-deficient mice was similar to that in IFN$y$-deficient mice, of which all died at day 9 (Fig 7B). Furthermore, wildtype mice recovered fully from the infection (Fig 7B). When parasite numbers in tissues of infected animals were measured, Irgm2deficient mice showed increased parasite loads in all tested tissues compared with wild-type mice (Fig 7C). Moreover, the parasite numbers in tissues of Irgm2-deficient mice were comparable with those in IFN- $y$-deficient mice (Fig S6A), which indicates that Irgm2 critically controls host defense against $T$. gondii infection.

\section{Discussion}

In the present study, we demonstrate that regulatory IRG Irgm2 plays a pivotal role in anti-T. gondii cell-autonomous immunity. At the molecular level, we characterized three features of Irgm2: the
GMS configuration of the N-terminal GTPase domain, the C-terminal Cys358 for PVM targeting, and ubiquitination on the lysine residues. The M77A mutation severely affected Irgm2-induced anti-T. gondii killing, recruitment of Irgb6 and Gbp1, and Irgm2 localization on the T. gondii PVM, which indicates that the GMS configuration of the $\mathrm{N}$-terminal GTPase domain plays a central role in Irgm2-mediated anti-T. gondii cell-autonomous immunity. The Irgm1 GMS mutant profoundly reduces affinity for GTP and impairs GTPase functions (Taylor et al, 1997; Martens et al, 2004). In addition, the GTPase domain of IRG proteins plays a role in dimerization of IRGs (Haldar et al, 2013). Considering that Irgm2 deficiency only affected Irgb6 recruitment, the GMS configuration of Irgm2 might be important for heterodimerization with Irgb6 through the N-terminal GTPase domain. In contrast to the M77A mutation, the C358A mutation adjacent to the C-terminal $\alpha \mathrm{K}$ specifically impaired Irgm2 localization on the T. gondii PVM. In the case of Irgm1, a tight cluster of Cys residues adjacent to the $\alpha \mathrm{K}$ is palmitoylated and important for its localization on host mitochondria and the Golgi apparatus (Henry et al, 2014). Although we do not have direct evidence of protein modification of Cys358 in Irgm2, it is plausible to postulate that modification on Cys358, including palmitoylation, might determine Irgm2 localization on the T. gondii PVM. Although the Irgm2 KA mutant localized at the T. gondii PVM, the percentages of parasites coated with ubiquitin at later time points in Irgm2 KA mutantreconstituted cells was comparable with those in C358A mutantreconstituted cells but significantly less than those in wild-type 

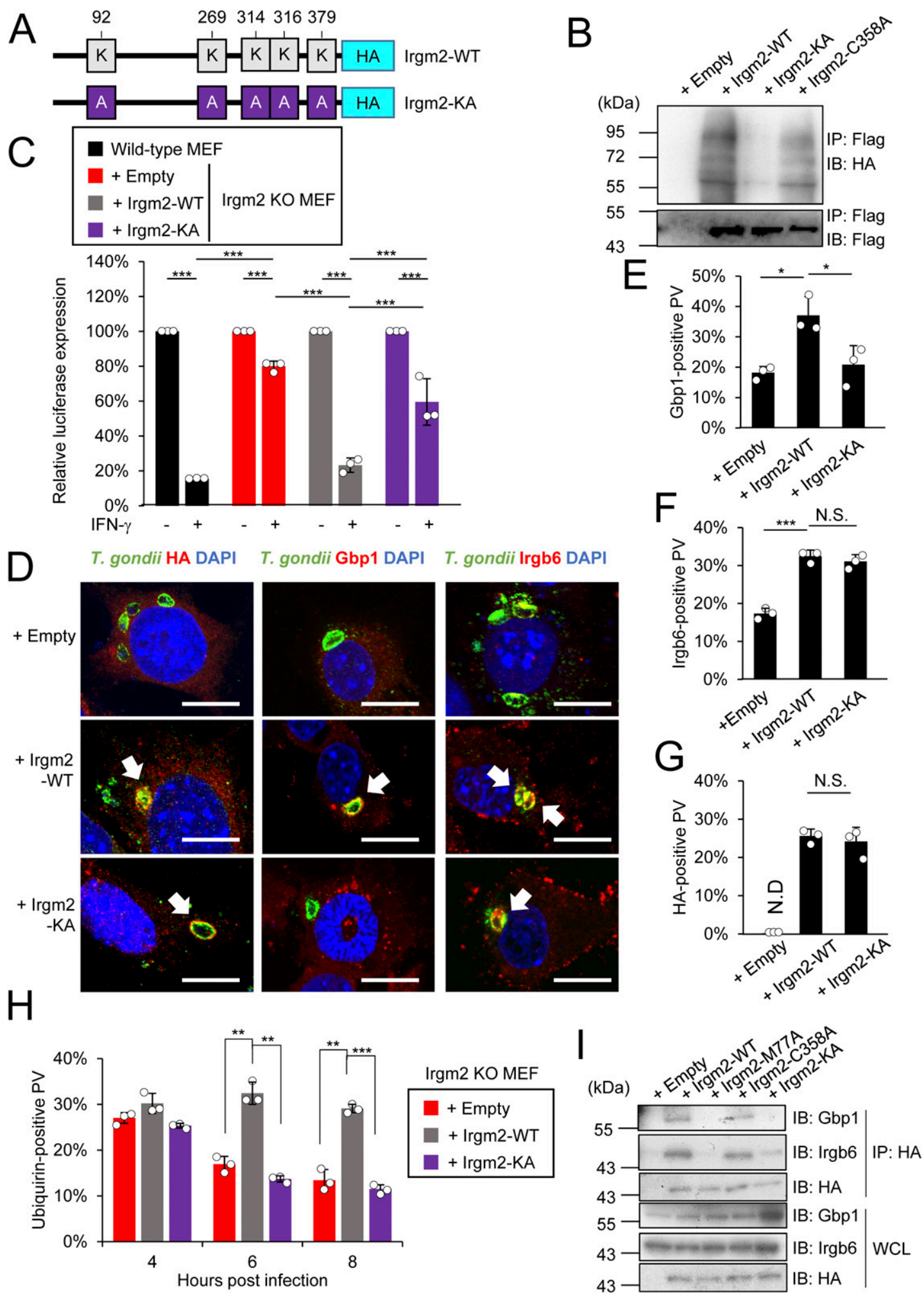

Figure 6. Irgm2 ubiquitination regulates Gbp1-mediated Toxoplasma gondii killing.

(A) Schematic overview of the substitution mutation site on the Irgm2 sequence. (B) Western blot image of showing ubiquitin-3xHA immunoprecipitated with Flag tagged Irgm2. Irgm2 KO MEFs stably expressing ubiquitin-3xHA and indicated Irgm2 variant. (C) T. gondii survival rate in the indicated Irgm2 reconstitution in Irgm2 KO MEFs with IFN- $y$ stimulation relative to those without IFN- $y$ treatment by luciferase analysis at $24 \mathrm{~h}$ post infection. (D) Confocal microscope images to show the localization of Irgm2-HA, Gbp1, and Irgb6 (red) to T. gondii parasitophorous vacuole (green), and DAPI (blue) at $4 \mathrm{~h}$ post infection in IFN- $\gamma$ treated Irgm2-KO MEFs reconstituted with indicated Irgm2 variants. (E, F, G) Percentages of recruitment of Gbp1 (E), Irgb6 (F), and HA (G). (H) T. gondii survival rate in the indicated Irgm2 reconstitution in Irgm2 KO 
A

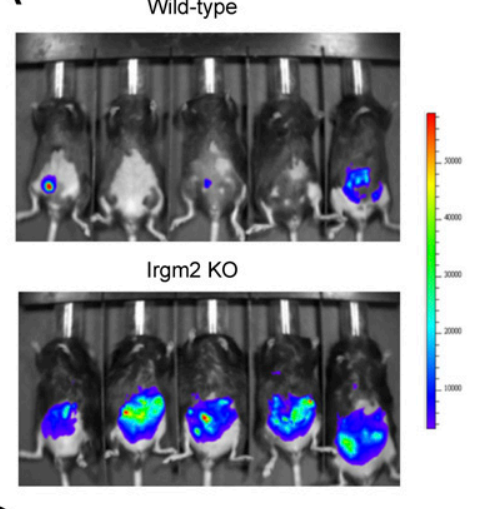

B

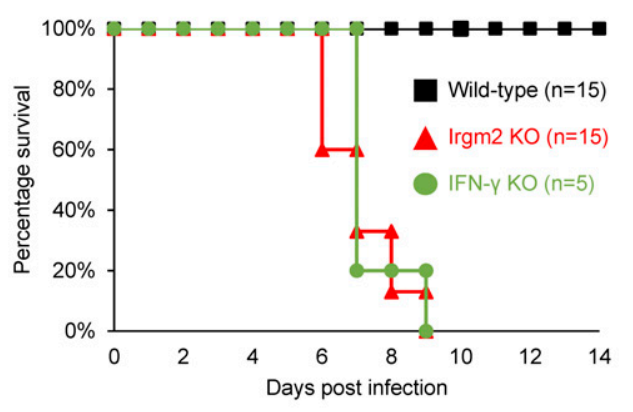

Figure 7. Irgm2 KO mice are highly susceptible to Toxoplasma gondii infection.

(A) In vivo bioluminescence imaging comparing between WT and Irgm2 KO mice on day 5 post infection. (B) Survival rate of T. gondii infected WT, IFN- $\gamma$ KO, and Irgm2 KO mice. (C) Parasite number in the various tissues collected from infected mice on day 5 post infection. (B, C) The data are combined results of three independent experiments (B, C). (A) Images are representative of three independent experiments (A). Survival duration comparison between two groups was analyzed by Kaplan-Meier survival analysis logrank test. Comparison of infection burden between WT versus Irgm2-KO was analyzed with Mann-Whitney Test. ${ }^{*} P<0.05,{ }^{* \star} P<0.01,{ }^{\star \star \star} P<0.001$.
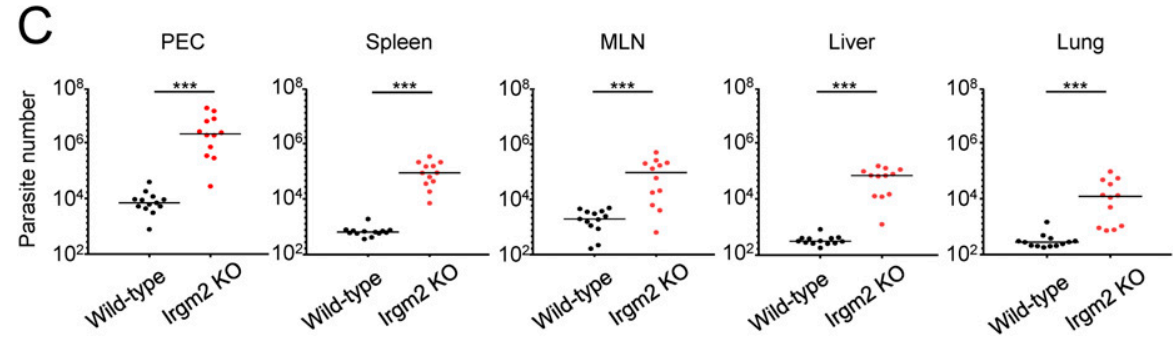

cells. However, Irgm2 KA mutant-reconstituted Irgm1/Irgm2/Irgm3 TKO cells exhibited recruitment of the KA mutant comparably with wild-type Irgm2 but showed no ubiquitination on the PVM, which suggests that Irgm2 itself is not ubiquitinated at the PVM. At present, the mechanism by which Irgm2 is ubiquitinated remains unclear. Future studies may reveal the molecular mechanism and responsible ubiquitin ligase. Moreover, considering that regulatory IRGs in uninfected cells localize at the Golgi or ER (Martens et al, 2005), Irgm2 might control other membrane trafficking events. We could not dissect the biological significance of Irgm2 localization on the $T$. gondii PVM in this study. Because Irgm2 localization on the T. gondii PVM was required for prolonged recruitment of p62 and ubiquitin to the vacuole without affecting parasite killing, it would be of interest to examine the role of Irgm2 localization on the T. gondii PVM in parasite killing-independent anti-T. gondii responses in the future.

Irgm2-deficient cells showed specific defects in recruitment of Irgb6 and Gbp1 but not Irga6 or Gbp2, which suggests selective requirement of Irgm2 in Irgb6- and Gbp1-dependent anti-T. gondii responses. Conversely, Irgm1 and Irgm3 globally control the anti-T. gondii cellular response that involves Irga6, Irgb6, Gbp1, and Gbp2 (Lee et al, 2015). Thus, Irgm2 uniquely participates in IFN-inducible GTPase-mediated cell-autonomous immunity against T. gondii. Considering that Irgm2 interacts with Irgb6 but not Irga6 (Hunn et al, 2008), a regulatory role of Irgm2 in the effector function of Irgb6 may be probable. However, defective Gbp1 recruitment in Irgm2deficient cells may be dependent or independent of Irgb6. It has been previously shown that Irgb6 controls Gbp1 accumulation on the T. gondii PVM (Lee et al, 2020), which suggests a direct link between Irgb6 and Gbp1.

The interaction of ubiquitinated Irgm2 with Gbp1 occurs in the cytosol. We found that Gbp1 loading on the PVM was decreased in ubiquitination-defective Irgm2 KA mutant-reconstituted Irgm2deficient cells. In addition, the KA Irgm2 mutant did not interact with Gbp1. The Irgm2 C358A mutant associated with Gbp1. In addition, reconstitution of the Irgm2 C358A mutant recovered the loading of Gbp1. The Irgm2 C358A mutant did not localize at the PVM or Golgi apparatus. Collectively, ubiquitinated Irgm2 must interact with Gbp1 to regulate Gbp1 recruitment to the T. gondii PVM in the cytosol but not at the PVM or Golgi apparatus. Irgm2 ubiquitination in the cytosol is required for Gbp1 recruitment to the PVM, its damage, and subsequent ubiquitination. The rapid reduction of ubiquitin accumulation in Irgm2 KA mutant-reconstituted cells may be not due to Irgm2 ubiquitination on the PVM but to an indirect effect by the failure of Gbp1-mediated PVM disruption.

Cells that lack Irgm1/Irgm3 or Atg proteins display severely impaired accumulation of effector IRGs, GBPs, ubiquitin, and p62 (Haldar et al, 2013; Ohshima et al, 2014; Lee et al, 2015), which indicates that regulatory IRGs and Atg proteins play a positive role in localizing effector proteins on the T. gondii PVM in IFN- $\gamma$-stimulated cells. In sharp contrast, neither regulatory IRGs nor Atg proteins control Irgm2 localization on the PVM. There is a coordinated loading mechanism of effector IRGs and GBPs on the T. gondii PVM

MEFs with IFN- $y$ stimulation relative to those without IFN- $y$ treatment by luciferase analysis at $24 \mathrm{~h}$ post infection. (I) Western blot image of showing Gbp1 and Irgb6 immunoprecipitated with HA tagged Irgm2 of the indicated variants stably expressed in Irgm2 KO MEFs. All graphs show the mean \pm SEM in three independent experiments. All images are representative of three independent experiments. N.D., not detected; ${ }^{*} P<0.05,{ }^{* *} P<0.01,{ }^{* * *} P<0.001$ IFN- $\gamma$-activated versus nonactivated and different antigen used in the co-culture used two-way ANOVA. Comparison between genotypes and different time points applied two-way ANOVA (Tukey's multiple comparisons test). White arrows indicate recruitment of effector on T. gondii parasitophorous vacuole. Scale bars on microscope images represent $10 \mu \mathrm{m}$. 
after formation (Khaminets et al, 2010; Lee et al, 2020). However, our current study indicates that Irgm2 is out of the temporal hierarchy that governs all other known IFN- $y$-inducible effectors for their localization on the T. gondii PVM. Considering that localization of reconstituted wild-type Irgm2 on the T. gondii PVM in Irgm2deficient cells requires IFN- $\gamma$ stimulation, unidentified IFN- $\gamma-$ inducible host factor(s) and upstream event(s) may be required for Irgm2 recruitment to the PVM.

Irgm2 is essential for anti-T. gondii host defense in vivo. Mice that lack Irgm2 were highly susceptible to $T$. gondii infection in a similar manner to IFN- $y$-deficient mice, which indicates a nonredundant essential function of Irgm2 in anti-T. gondii host defense. Compared with other regulatory IRGS, considering that the time course of death of Irgm2-deficient mice after T. gondii infection was similar to that of Irgm3/iNOS double-deficient mice (Zhao et al, 2009), Irgm2-deficient mice might be more susceptible to $T$. gondii than Irgm3-deficient mice in vivo. We found that Irgm2 deficiency led to partially defective translocation of Gbp1 and Irgb6 or reduced retention of p62 and ubiquitin on the T. gondii PVM. However, considering that Gbp1deficient mice have a relatively moderate increase in their in vivo susceptibility to T. gondii (Selleck et al, 2013), the partial defects in Gbp1 recruitment to the $T$. gondii PVM by Irgm2 deficiency might not be sufficient to explain the severe in vivo phenotype of Irgm2-deficient mice. Although additional defects in the recruitment of Irgb6, p62, and ubiquitin to the T. gondii PVM may account for the severe phenotype of Irgm2-deficient mice, whether the correlative reduction of IFN-inducible effector recruitment to the T. gondii PVM could strictly connect with the causation of the in vivo phenotype should be carefully examined in the future. For example, Irgm2 may have pleiotropic functions such as membrane trafficking at the Golgi. Moreover, recent studies have demonstrated a role for Irgm2 in controlling LPS-mediated caspase-11 activation (Eren et al, 2020; Finethy et al, 2020). Although the role of caspase-11 in T. gondii infection in vivo remains unknown, dysregulated caspase-11 activation and defective membrane trafficking might affect the high mortality of Irgm2-deficient mice infected with T. gondii. In summary, we have demonstrated that Irgm2 plays an important role in Gbp1/Irgb6-mediated parasite killing in IFN- $y$-induced cell-autonomous anti-T. gondii immunity.

\section{Materials and Methods}

\section{Cells, mice, and parasites}

Primary MEFs were maintained in DMEM (Nacalai Tesque) supplemented with $10 \%$ heat-inactivated FBS (Gibco, Life Technologies), 100 $\mathrm{U} / \mathrm{ml}$ penicillin (Nacalai Tesque), and $100 \mu \mathrm{g} / \mathrm{ml}$ streptomycin (Nacalai Tesque). MEFs that lack Irgm1/Irgm3, Atg proteins, Irgb6, and Gbp1 are described previously (Lee et al, 2015, 2020; Sasai et al, 2017). Bone marrow-derived macrophages were generated by cultivating BM progenitors isolated from BM in complete medium containing $10 \%$ L-cell conditioned medium for $6-7 \mathrm{~d}$. The complete medium comprised 10\% heat-inactivated FBS in RPMI 1640 medium (Nacalai Tesque). T. gondii were parental Pru $\triangle \mathrm{HX}$, luciferase-expressing Pru $\triangle \mathrm{HX}$, and OVAexpressing Pru $\triangle \mathrm{HX}$. They were maintained in Vero cells by passaging every 3 d in RPMI 1640 supplemented with 2\% heat-inactivated FBS, 100
$\mathrm{U} / \mathrm{ml}$ penicillin, and $100 \mu \mathrm{g} / \mathrm{ml}$ streptomycin. All animal experiments were conducted with approval of the Animal Research Committee of Research Institute for Microbial Diseases in Osaka University.

\section{Generation of Irgm2-deficient mice by CRISPR/Cas9 genome editing}

The target gRNA sequence of Irgm2 was 5'-gagaaagattcagctcccacTGG-3' (TGG; the PAM sequence) in the $\mathrm{N}$-terminus. The insert fragments of Irmg2 gRNA were generated using the primers Irgm2_gRNA_F 5'TTAATACGACTCACTATAGGgagaaagattcagctcccacGTTTAGAGCTAGAAATAGCAAGTTAAAAT-3' and gRNA_R 5'-AAAAGCACCGACTCGGTGCCACTTाTCAAGTTGATAACGGACTAGCCTTATTTAACTTGCTATTTCTAGCTCT-3'. T7-transcribed Irgm2 gRNA PCR products were purified in gels and used for subsequent generation of gRNA. MEGAshortscript 7 (Life Technologies) was used to generate the gRNA. mRNA that encoded RNA-guided DNA endonuclease Cas9 was generated by in vitro transcription using the mMESSAGE mMACHINE T7 ULTRA kit (Life Technologies). The template was amplified by PCR using pEF6-hCas9-Puro and primers T7Cas9_IVT_F and Cas9_R, and then purified in a gel. The synthesized gRNA and Cas9 mRNA were purified using the MEGAclear kit (Life Technologies) and eluted in RNase-free water (Nacalai Tesque). To generate Irgm2-deficient mice, 6 -wk-old female C57BL/ 6 mice were superovulated and mated with C57BL/ 6 males. Fertilized one-cell-stage embryos were collected from the oviducts and Cas9-encoding mRNA (100 ng/ $\mu \mathrm{l}$ ) and gRNA (50 $\mathrm{ng} / \mu \mathrm{l})$ were injected into the cytoplasm as described previously (Sasai et al, 2017). The injected live embryos were transferred into the oviducts of pseudopregnant Institute of Cancer Research females at $0.5 \mathrm{~d}$ post-coitus. Heterozygous mice were intercrossed to generate Irgm2-deficient mice for use in the in vivo experiments. Irgm2deficient mice were born at Mendelian ratios and were healthy. Expression of Irgm2 proteins in primary embryonic fibroblasts was analyzed by Western blotting. Two embryos were used to generate two independent Irgm2-deficient MEF lines that similarly showed defects in anti-T. gondii responses (data not shown). Irgm1/Irgm2/ Irgm3-TKO mice were generated through genome editing by introducing the same Irgm2-targeting gRNA used to generate Irgm2-deficient mice as described above together with Irgm1- or Irgm3-targeting gRNAs into mouse embryos (Lee et al, 2015). Irgm1/Irgm2/Irgm3-TKO mice were born at Mendelian ratios and were healthy. Expression of Irgm1, Irgm2, and Irgm3 proteins in primary embryonic fibroblasts was analyzed by Western blotting (Fig S6B).

\section{Cloning and recombinant expression}

The region of interest of the CDNA corresponding to the wild-type Irgm2 or indicated point mutants or deletion mutants of Irgm2 (GenBank accession no. NM_019440.3) was synthesized from the mRNA of the spleen of C57BL6 mice using primers Irgm2_F 5'-gaattcaccATGGAAGAGGCAGTTGAGTCACCTGAG-3' and Irgm2_R 5'-ctcgagAGGATGAGGAATGGAGAGTCTCAG-3'. Irgm2 GMS, C357A, and C358A mutants were generated using primers GMS_F 5'-CTGGGGACTCTGGCAATGGCgCgTCATCTTTCATCAATGCCCT-3' and GMS_R 5'-AGGGCATTGATGAAAGATGACgCGCCATTGCCAGAGTCCCCAG-3'; C357A_F 5'-TAGGTTाTGACTACATGAAGgCgTGCTTACCTCTCATCACAG-3' and C357A_R 5'-CTGTGATGAGAGGTAAAGCAcgcCTTCATGTAGTCAAAACCTA-3'; C358A_F 5'-GTTाGACTACATGAAGTGCgCCTTTACCTCTCATCACAGTCG-3' and C358A_R 
5'-CGACTGTGATGAGAGGTAAAggcGCACTTCATGTAGTCAAAAC-3'. KA mutants of Irgm2 were artificially synthesized (FASMAC). PCR products were ligated into the ECoRI/Xhol site of the retroviral PMRX-HA expression vector for retroviral infection. The sequences of all constructs were confirmed by DNA sequencing.

\section{Mice survival and in vivo parasite imaging}

Mice were intraperitoneally infected with Pru $\Delta \mathrm{HX}$ T. gondii tachyzoites that expressed luciferase ( $1 \times 10^{4}$ in $200 \mu \mathrm{l}$ PBS per mouse). Mice survival was monitored for up to $15 \mathrm{~d}$ postinfection. For in vivo imaging of parasites, mice were intraperitoneally injected with $3 \mathrm{mg}$ D-luciferin in $200 \mu \mathrm{l}$ PBS (Promega) on day 5 postinfection. Mice were subjected to inhalation anesthesia by isoflurane (Sumitomo Dainippon Pharma). Abdominal photon emission was assessed during 60 s of exposure by an in vivo imaging system (IVIS Spectrum; Xenogen), followed by analysis with Living Image software (Xenogen).

\section{Reagents}

Antibodies against Irgb6 (T-cell specific GTPase [TGTP]; Sc-11079), Irgm1 (LRG-47; SC-11075), Irgm2 (GTPI; Sc-11088), and Irgm3 (inducibly expressed GTPase [IGTP]; sc-136317) were purchased from Santa Cruz Biotechnology, Inc. Antibodies against FLAG M2 (F3165), $\beta$-actin (A1978), and HA were obtained from Sigma-Aldrich. An Anti-HA 1.1 mouse monoclonal antibody was purchased from BioLegend. Rabbit polyclonal anti-GBP2 and mouse monoclonal anti-p62 (PM045) antibodies were obtained from Proteintech and MBL International, respectively. An anti-ubiquitin mouse monoclonal antibody (FK2; MFK-004) was obtained from Nippon Biotest Laboratories. A mouse monoclonal anti-Irga6 (10D7) antibody was provided by Dr. JC Howard (Instituto Gulbenkian de Ciencia). A rabbit polyclonal anti-GBP1 antibody was provided by Dr JC Boothroyd (Stanford University School of Medicine). A mouse monoclonal antiGRA2 antibody were provided by Dr. D Soldati-Favre (University of Geneva). A custom anti-Irgm2 antibody was purchased from Cosmobio for microscopy analysis. Recombinant mouse IFN- $y$ was purchased from PeproTech.

\section{Western blotting}

MEFs and macrophages were stimulated with IFN- $\gamma(10 \mathrm{ng} / \mathrm{ml})$ overnight. The cells were washed with PBS and then lysed with 1× (TNE) Tris/NP-40/EDTA buffer (20 mM Tris-HCl, $150 \mathrm{mM} \mathrm{NaCl}$, $1 \mathrm{mM}$ EDTA, and 1\% NP-40) or Onyx buffer (20 mM Tris- $\mathrm{HCl}, 135 \mathrm{mM}$ $\mathrm{NaCl}, 1 \%$ Triton- $X$, and $10 \%$ glycerol) for immunoprecipitation, which contained a protease inhibitor cocktail (Nacalai Tesque) and sonicated for $30 \mathrm{~s}$. The supernatant was collected, incubated with the relevant antibodies overnight, and then pulled down with Protein G Sepharose (GE) for immunoprecipitation. Samples and/or total protein was loaded and separated in $10 \%$ or $15 \%$ SDS-PAGE gels. After the appropriate length was reached, the proteins in the gel were transferred to a polyvinyl difluoride membrane. The membranes were blocked with 5\% dry skim milk (BD Difco Skim milk) in PBS/Tween 20 (0.2\%) at room temperature. The membranes were probed overnight at $4^{\circ} \mathrm{C}$ with the indicated primary antibodies. After washing with PBS/Tween, the membranes were probed with HRP-conjugated secondary antibodies for $1 \mathrm{~h}$ at room temperature and then visualized by Luminata Forte Western HRP substrate (Millipore).

\section{Measurement of T. gondii numbers by a luciferase assay}

To measure the number of $T$. gondii, cells were untreated or treated with IFN- $\gamma(10 \mathrm{ng} / \mathrm{ml})$ for $24 \mathrm{~h}$. After the stimulation, the cells were infected with luciferase-expressing Pru $\Delta \mathrm{HX}$ T. gondii (MOI of 0.5 ) for $24 \mathrm{~h}$. The infected cells were collected and lysed with $100 \mu \mathrm{l}$ of $1 \times$ passive lysis buffer (Promega). The samples were sonicated for $30 \mathrm{~s}$ before centrifugation and $5 \mu \mathrm{l}$ of the supernatants were collected for luciferase expression reading by the dual-luciferase reporter assay system (Promega) using a GLOMAX 20/20 luminometer (Promega). The in vitro data are presented as the percentage of $T$. gondii survival in IFN- $y$-stimulated cells relative to unstimulated cells (control).

To measure the number of $T$. gondii in the peritoneal cavity, mesenteric lymph nodes, spleen, liver, and lungs, these organs were removed on day 5 post-infection. The samples were homogenized and lysed in $1 \mathrm{ml}$ of $1 \times$ passive lysis buffer, followed by sonication for $30 \mathrm{~s}$. After centrifugation, luciferase activity was measured using $5 \mu \mathrm{l}$ of the supernatants collected for luciferase expression reading as described for the in vitro model. The in vivo data are presented as absolute values.

\section{Immunofluorescence microscopy}

MEFs were infected with T. gondii (MOI 5 or 2 ) after stimulation with IFN- $\gamma(10 \mathrm{ng} / \mathrm{ml})$ for $24 \mathrm{~h}$. The cells were infected for the indicated time in the respective figures and then fixed for $10 \mathrm{~min}$ in PBS containing 3.7\% formaldehyde. Cells were then permeabilized with PBS containing $0.002 \%$ digitonin (Nacalai Tesque) and blocked with $8 \%$ FBS in PBS for $1 \mathrm{~h}$ at room temperature. Next, the cells were incubated with antibodies relevant to the experiments for $1 \mathrm{~h}$ at $37^{\circ} \mathrm{C}$. After gently washing the samples in PBS, the samples were incubated with Alexa 488- and 594-conjugated secondary antibodies as well as DAPI for $1 \mathrm{~h}$ at $37^{\circ} \mathrm{C}$ in the dark. The samples were then mounted onto glass slides with PermaFluor (Thermo Fisher Scientific) and observed under a confocal laser microscope (FV1200 IX-83; Olympus). Images are shown at $\times 1,000$ magnification (scale bar at 5 or $10 \mu \mathrm{m}$ as indicated). To measure recruitment rates, 100 vacuoles were observed and the numbers of vacuoles coated with effectors were calculated. The counting was repeated three times (three technical replicates). The mean of the three technical replicates was calculated and shown in each circle. After the independent experiments were repeated three times (three biological replicates), three means (three circles) are shown in each figure.

\section{Mass spectrometric analysis}

MEFs that expressed Spot-tagged Irgm2 were lysed in radioimmunoprecipitation assay (RIPA) buffer (20 mM Hepes- $\mathrm{NaOH}, \mathrm{pH}$ 7.5, $1 \mathrm{mM}$ EGTA, $1 \mathrm{mM} \mathrm{MgCl}$, $150 \mathrm{mM} \mathrm{NaCl}, 0.25 \%$ sodium deoxycholate, $0.05 \%$ SDS, and $1 \%$ NP-40) containing Complete protease and PhosSTOP phosphatase inhibitors (Roche). The lysates were 
incubated with anti-Spot nanobody-coupled magnetic agarose beads (Spot-trap_MA; ChromoTek) at $4^{\circ} \mathrm{C}$ for $3 \mathrm{~h}$. The beads were washed four times with RIPA buffer and then twice with $50 \mathrm{mM}$ ammonium bicarbonate. Proteins on the beads were digested with $200 \mathrm{ng}$ trypsin (MS grade; Thermo Fisher Scientific) at $37^{\circ} \mathrm{C}$ for $16 \mathrm{~h}$. The digests were reduced, alkylated, acidified, and desalted with GLTip SDB (GL Sciences). The eluates were evaporated and dissolved in 3\% acetonitrile (ACN) and $0.1 \%$ trifluoroacetic acid. LC-MS/MS analysis of the resultant peptides was performed on an EASY-nLC 1200 UHPLC connected to a $\mathrm{Q}$ Exactive Plus mass spectrometer equipped with a nanoelectrospray ion source (Thermo Fisher Scientific). The peptides were separated on a $75 \mu \mathrm{m}$ inner diameter $\times 150 \mathrm{~mm}$ C18 reversed-phase column (Nikkyo Technos) with a linear 4-32\% ACN gradient for 0-100 min, followed by an increase to $80 \%$ ACN for 10 min. The mass spectrometer was operated in data-dependent acquisition mode with the top $10 \mathrm{MS} / \mathrm{MS}$ method. MS1 spectra were measured with a resolution of 70,000 , an automatic gain control target of $1 \times 10^{6}$, and a mass range from 350 to $1,500 \mathrm{~m} / \mathrm{z}$. HCD MS/MS spectra were acquired at a resolution of 17,500 , an automatic gain control target of $5 \times 10^{4}$, an isolation window of $2.0 \mathrm{~m} / \mathrm{z}$, a maximum injection time of $60 \mathrm{~ms}$, and a normalized collision energy of 27. Dynamic exclusion was set to $10 \mathrm{~s}$. Raw data were directly analyzed against the NCBI nonredundant database restricted to Mus musculus using Proteome Discoverer v2.3 (Thermo Fisher Scientific) with the Sequest HT search engine. The search parameters were as follows: (a) trypsin as an enzyme with up to two missed cleavages; (b) precursor mass tolerance of $10 \mathrm{ppm}$; (c) fragment mass tolerance of $0.02 \mathrm{D}$; (d) carbamidomethylation of cysteine as a fixed modification; (e) acetylation of the protein $\mathrm{N}$-terminus, oxidation of methionine, di-glycine modification of lysine and phosphorylation of serine, threonine, and tyrosine as variable modifications. Peptides were filtered at a false discovery rate of $1 \%$ using the percolator node.

\section{Statistical analysis}

Three points in all graphs represent three means derived from three independent experiments (three biological replicates). All statistical analyses were performed using Prism 7 (GraphPad). In infection assays, differences in the $T$. gondii inhibition activity between IFN- $y$ activated versus nonactivated were subjected to two-way ANOVA with Tukey's multiple comparisons test to analyze the difference between genotypes. When comparing effector recruitment percentages between different genotypes, ordinary oneway ANOVA was used when there were more than two groups. If there were only two groups, the Mann-Whitney test was applied instead. In cases where the effector recruitment percentage was compared with consideration that it was affected by more than one variable, for example genotype and different time points, the adopted analysis was two-way ANOVA (Tukey's multiple comparisons test). Statistical significance of the difference in the survival of mice between two groups was assessed by Kaplan-Meier survival analysis and the log-rank test.

\section{Supplementary Information}

Supplementary Information is available at https://doi.org/10.26508/lsa. 202000960.

\section{Acknowledgements}

We thank M Enomoto (Osaka University) for secretarial assistance. This study was supported by the Research Program on Emerging and Re-emerging Infectious Diseases (JP20fk0108137), Japanese Initiative for Progress of Research on Infectious Diseases for Global Epidemic (JP20wm0325010) and the Strategic International Collaborative Research Program (JP20jm0210067) from the Agency for Medical Research and Development (AMED), a Grant-inAid for Transformative Research Area (B) (Establishment of PLAMP as a new concept to determine self and nonself for obligatory intracellular pathogens; 20B304), for Scientific Research on Innovation Areas (production, function and structure of neo-self; 19H04809), for Scientific Research (B) (18KK0226 and 18H02642) and for Scientific Research (A) (19H00970) from the Ministry of Education, Culture, Sports, Science and Technology, Fusion Oriented Research for Disruptive Science and Technology (JPMJFR206D) and Moonshot research \& development (JPMJMS2025) from Japan Science and Technology Agency, program from Joint Usage and Joint Research Programs of the Institute of Advanced Medical Sciences, Tokushima University, Takeda Science Foundation, Mochida Memorial Foundation, Astellas Foundation for Research on Metabolic Disorders, and Research Foundation for Microbial Diseases of Osaka University.

\section{Author Contributions}

A Pradipta: conceptualization, data curation, formal analysis, validation, investigation, methodology, and writing-original draft, review, and editing

M Sasai: data curation, formal analysis, and investigation K Motani: data curation, investigation, and methodology. JS Ma: formal analysis, investigation, and methodology.

Y Lee: investigation and methodology.

$\mathrm{H}$ Kosako: formal analysis, investigation, and methodology. M Yamamoto: conceptualization, resources, data curation, formal analysis, supervision, funding acquisition, investigation, project administration, and writing-original draft, review, and editing.

\section{Conflict of Interest Statement}

The authors declare that they have no conflict of interest.

\section{References}

Bekpen C, Hunn JP, Rohde C, Parvanova I, Guethlein L, Dunn DM, Glowalla E, Leptin M, Howard JC (2005) The interferon-inducible p47 (IRG) GTPases in vertebrates: Loss of the cell autonomous resistance mechanism in the human lineage. Genome Biol 6: R92. doi:10.1186/gb-2005-6-11-r92

Choi J, Park S, Biering SB, Selleck E, Liu CY, Zhang X, Fujita N, Saitoh T, Akira S, Yoshimori T, et al (2014) The parasitophorous vacuole membrane of toxoplasma gondii is targeted for disruption by ubiquitin-like conjugation systems of autophagy. Immunity 40: 924-935. doi:10.1016/ j.immuni.2014.05.006

Eren E, Planès R, Bagayoko S, Bordignon PJ, Chaoui K, Hessel A, Santoni K, Pinilla M, Lagrange B, Burlet-Schiltz O, et al (2020) Irgm2 and Gate-16 cooperatively dampen Gram-negative bacteria-induced caspase-11 response. EMBO Rep 21: e50829. doi:10.15252/embr.202050829

Finethy R, Dockterman J, Kutsch M, Orench-Rivera N, Wallace GD, Piro AS, Luoma S, Haldar AK, Hwang S, Martinez J, et al (2020) Dynamin-related Irgm proteins modulate LPS-induced caspase-11 activation and septic shock. EMBO Rep 21: e50830. doi:10.15252/embr.202050830 
Gazzinelli RT, Hakim FT, Hieny S, Shearer GM, Sher A (1991) Synergistic role of $\mathrm{CD} 4+$ and $\mathrm{CD} 8+\mathrm{T}$ lymphocytes in IFN-gamma production and protective immunity induced by an attenuated Toxoplasma gondii vaccine. J Immunol 146: 286-292.

Haldar AK, Foltz C, Finethy R, Piro AS, Feeley EM, Pilla-Moffett DM, Komatsu M, Frickel EM, Coers J (2015) Ubiquitin systems mark pathogencontaining vacuoles as targets for host defense by guanylate binding proteins. Proc Natl Acad Sci U S A 112: E5628-E5637. doi:10.1073/ pnas. 1515966112

Haldar AK, Saka HA, Piro AS, Dunn JD, Henry SC, Taylor GA, Frickel EM, Valdivia RH, Coers I (2013) IRG and GBP host resistance factors target aberrant, "non-self" vacuoles characterized by the missing of "self" IRGM proteins. PLoS Pathog 9: e1003414. doi:10.1371/journal.ppat.1003414

Henry SC, Schmidt EA, Fessler MB, Taylor GA (2014) Palmitoylation of the immunity related GTPase, Irgm1: Impact on membrane localization and ability to promote mitochondrial fission. PLoS One 9: e95021. doi:10.1371/journal.pone.0095021

Hunn JP, Koenen-Waisman S, Papic N, Schroeder N, Pawlowski N, Lange R, Kaiser F, Zerrahn J, Martens S, Howard JC (2008) Regulatory interactions between IRG resistance GTPases in the cellular response to Toxoplasma gondii. EMBO J 27: 2495-2509. doi:10.1038/ emboj.2008.176

Hunter CA, Remington JS (1995) The role of IL12 in toxoplasmosis. Res Immunol 146: 546-552. doi:10.1016/0923-2494(96)83030-6

Hunter CA, Sibley LD (2012) Modulation of innate immunity by Toxoplasma gondii virulence effectors. Nat Rev Microbiol 10: 766-778. doi:10.1038/ nrmicro2858

Khaminets A, Hunn JP, Könen-Waisman S, Zhao YO, Preukschat D, Coers J, Boyle JP, Ong YC, Boothroyd JC, Reichmann G, et al (2010) Coordinated loading of IRG resistance GTPases on to the Toxoplasma gondii parasitophorous vacuole. Cell Microbiol 12: 939-961. doi:10.1111/j.14625822.2010.01443.x

Kim BH, Shenoy AR, Kumar P, Bradfield CJ, MacMicking JD (2012) IFN-inducible GTPases in host cell defense. Cell Host Microbe 12: 432-444. doi:10.1016/j.chom.2012.09.007

Kravets E, Degrandi D, Ma Q, Peulen TO, Klümpers V, Felekyan S, Kühnemuth R, Weidtkamp-Peters S, Seidel CA, Pfeffer K (2016) Guanylate binding proteins (GBPs) directly attack Toxoplasma gondii via supramolecular complexes. Elife 5: e11479. doi:10.7554/eLife.11479

Kresse A, Konermann C, Degrandi D, Beuter-Gunia C, Wuerthner J, Pfeffer K, Beer S (2008) Analyses of murine GBP homology clusters based on in silico, in vitro and in vivo studies. BMC Genomics 9: 158. doi:10.1186/ 1471-2164-9-158

Lee Y, Sasai M, Ma JS, Sakaguchi N, Ohshima J, Bando H, Saitoh T, Akira S, Yamamoto M (2015) P62 plays a specific role in interferon- $y$-induced presentation of a toxoplasma vacuolar antigen. Cell Rep 13: 223-233. doi:10.1016/j.celrep.2015.09.005

Lee Y, Yamada H, Pradipta A, Ma JS, Okamoto M, Nagaoka H, Takashima E, Standley DM, Sasai M, Takei K, et al (2020) Initial phospholipiddependent Irgb6 targeting to Toxoplasma gondii vacuoles mediates host defense. Life Sci Alliance 3: e201900549. doi:10.26508/ Isa.201900549

Man SM, Karki R, Sasai M, Place DE, Kesavardhana S, Temirov J, Frase S, Zhu Q, Malireddi SRKS, Kuriakose T, et al (2016) IRGB10 liberates bacterial ligands for sensing by the AIM2 and caspase-11-NLRP3 inflammasomes. Cell 167: 382-396.e17. doi:10.1016/j.cell.2016.09.012

Maric-Biresev J, Hunn JP, Krut O, Helms JB, Martens S, Howard JC (2016) Loss of the interferon- $y$-inducible regulatory immunity-related GTPase (IRG), Irgm1, causes activation of effector IRG proteins on lysosomes, damaging lysosomal function and predicting the dramatic susceptibility of Irgm1-deficient mice to infection. BMC Biol 14: 33. doi:10.1186/s12915-016-0255-4
Martens S, Howard I (2006) The interferon-inducible GTPases. Annu Rev Cell Dev Biol 22: 559-589. doi:10.1146/annurev.cellbio.22.010305.104619

Martens S, Parvanova I, Zerrahn J, Griffiths G, Schell G, Reichmann G, Howard JC (2005) Disruption of Toxoplasma gondii parasitophorous vacuoles by the mouse p47-resistance GTPases. PLoS Pathog 1: e24. doi:10.1371/ journal.ppat.0010024

Martens S, Sabel K, Lange R, Uthaiah R, Wolf E, Howard JC (2004) Mechanisms regulating the positioning of mouse p47 resistance GTPases LRG-47 and IIGP1 on cellular membranes: Retargeting to plasma membrane induced by phagocytosis. J Immunol 173: 2594-2606. doi:10.4049/ jimmunol.173.4.2594

Mukhopadhyay D, Sangaré LO, Braun L, Hakimi MA, Saeij JP (2020) Toxoplasma GRA 15 limits parasite growth in IFN $\gamma$-activated fibroblasts through TRAF ubiquitin ligases. EMBO / 39: e103758. doi:10.15252/embj.2019103758

Ohshima J, Lee Y, Sasai M, Saitoh T, Su Ma J, Kamiyama N, Matsuura Y, Pann-Ghill S, Hayashi M, Ebisu S, et al (2014) Role of mouse and human autophagy proteins in IFN- $\gamma$-Induced cell-autonomous responses against toxoplasma gondii. J Immunol 192: 3328-3335. doi:10.4049/jimmunol.1302822

Ohshima J, Sasai M, Liu J, Yamashita K, Ma JS, Lee Y, Bando H, Howard JC, Ebisu S, Hayashi M, et al (2015) RabGDI $\alpha$ is a negative regulator of interferon$\gamma$-inducible GTPase-dependent cell-autonomous immunity to Toxoplasma gondii. Proc Natl Acad Sci U S A 112: E4581-E4590. doi:10.1073/pnas.1510031112

Papic N, Hunn JP, Pawlowski N, Zerrahn J, Howard JC (2008) Inactive and active states of the interferon-inducible resistance GTPase, Irga6, in vivo. J Biol Chem 283: 32143-32151. doi:10.1074/jbc.M804846200

Park S, JongSoo L, Park SH, Yu HS, Lee J (2016) Targeting by AutophaGy proteins (TAG): Targeting of IFNG-inducible GTPases to membranes by the LC3 conjugation system of autophagy. Autophagy 12: 1153-1167. doi:10.1080/15548627.2016.1178447

Pilla-Moffett D, Barber MF, Taylor GA, Coers J (2016) Interferon-inducible GTPases in host resistance, inflammation and disease. J Mol Biol 428: 3495-3513. doi:10.1016/j.jmb.2016.04.032

Saeij JP, Frickel EM (2017) Exposing toxoplasma gondii hiding inside the vacuole: A role for GBPs, autophagy and host cell death. Curr Opin Microbiol 40: 72-80. doi:10.1016/j.mib.2017.10.021

Sasai M, Sakaguchi N, Ma JS, Nakamura S, Kawabata T, Bando H, Lee Y, Saitoh T, Akira S, Iwasaki A, et al (2017) Essential role for GABARAP autophagy proteins in interferon-inducible GTPase-mediated host defense. Nat Immunol 18: 899-910. doi:10.1038/ni.3767

Selleck EM, Fentress SJ, Beatty WL, Degrandi D, Pfeffer K, Virgin HW 4th, Macmicking JD, Sibley LD (2013) Guanylate-binding protein 1 (Gbp1) contributes to cell-autonomous immunity against toxoplasma gondii. PLoS Pathog 9: e1003320. doi:10.1371/journal.ppat.1003320

Sturge CR, Yarovinsky F (2014) Complex immune cell interplay in the gamma interferon response during Toxoplasma gondii infection. Infect Immun 82: 3090-3097. doi:10.1128/IAI.01722-14

Taylor GA, Collazo CM, Yap GS, Nguyen K, Gregorio TA, Taylor LS, Eagleson B, Secrest L, Southon EA, Reid SW, et al (2000) Pathogen-specific loss of host resistance in mice lacking the IFN- $y$-inducible gene IGTP. Proc Natl Acad Sci U S A 97: 751-755. doi:10.1073/pnas.97.2.751

Taylor GA, Stauber R, Rulong S, Hudson E, Pei V, Pavlakis GN, Resau JH, Vande Woude GF (1997) The inducibly expressed GTPase localizes to the endoplasmic reticulum, independently of GTP binding. I Biol Chem 272: 10639-10645. doi:10.1074/jbc.272.16.10639

Tiwari S, Choi HP, Matsuzawa T, Pypaert M, MacMicking JD (2009) Targeting of the GTPase Irgm1 to the phagosomal membrane via PtdIns(3,4)P2 and PtdIns(3,4,5)P3 promotes immunity to mycobacteria. Nature Immunol 10: 907-917. doi:10.1038/ni.1759

Uthaiah RC, Praefcke GJ, Howard JC, Herrmann C (2003) IIGP1, an interferon$\gamma$-inducible $47-\mathrm{kDa}$ GTPase of the mouse, showing cooperative enzymatic activity and GTP-dependent multimerization. J Biol Chem 278: 29336-29343. doi:10.1074/jbc.M211973200 
Yamamoto M, Okuyama M, Ma JS, Kimura T, Kamiyama N, Saiga H, Ohshima J, Sasai M, Kayama H, Okamoto T, et al (2012) A cluster of interferon$\gamma$-inducible p65 gtpases plays a critical role in host defense against toxoplasma gondii. Immunity 37: 302-313. doi:10.1016/ j.immuni.2012.06.009

Yarovinsky F, Sher A (2006) Toll-like receptor recognition of Toxoplasma gondii. Int J Parasitol 36: 255-259. doi:10.1016/j.ijpara.2005.12.003

Zhao Y, Ferguson DJ, Wilson DC, Howard JC, Sibley LD, Yap GS (2009) Virulent toxoplasma gondii evade immunity-related GTPase-mediated parasite vacuole disruption within primed macrophages. I Immunol 182: 3775-3781. doi:10.4049/jimmunol.0804190

Zhao YO, Könen-Waisman S, Taylor GA, Martens S, Howard JC (2010) Localisation and mislocalisation of the interferon-inducible immunity-related GTPase, Irgm1 (LRG-47) in mouse cells. PLoS One 5: e8648. doi:10.1371/journal.pone.0008648

Zhao Z, Fux B, Goodwin M, Dunay IR, Strong D, Miller BC, Cadwell K, Delgado MA, Ponpuak M, Green KG, et al (2008) Autophagosome-independent essential function for the autophagy protein Atg5 in cellular immunity to intracellular pathogens. Cell Host Microbe 4: 458-469. doi:10.1016/ j.chom.2008.10.003

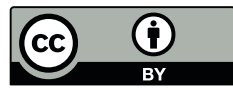

License: This article is available under a Creative Commons License (Attribution 4.0 International, as described at https://creativecommons.org/ licenses/by/4.0/). 